\title{
Weight loss as a predictor of mortality in patients with interstitial lung disease
}

To the Editor:

The interstitial lung diseases (ILDs) are a diverse group of diffuse parenchymal lung disorders that commonly result in pulmonary fibrosis. ILDs are broadly classified according to known and unknown aetiologies. Connective tissue disease-associated ILD (CTD-ILD) and chronic hypersensitivity pneumonitis (CHP) are among the most common ILDs of known aetiology, while idiopathic pulmonary fibrosis (IPF) and unclassifiable ILD (U-ILD) are among the most common of unknown aetiology [1-4].

There exists substantial heterogeneity in natural history between ILD subtypes, but clinical characteristics such as age, gender and pulmonary function have been shown to predict mortality across ILD subtypes [5]. Baseline body mass index (BMI) has also been linked to outcomes in IPF [6], but the clinical significance of longitudinal changes in BMI has not been reported. We hypothesised that weight loss would be associated with worse outcome in patients with common forms of ILD.

We conducted a retrospective cohort study (IRB approved protocol \#875917) of patients with ILD at the University of California at Davis. Consecutive consenting patients with a multi-disciplinary diagnosis of IPF, CTD-ILD, CHP or U-ILD and at least two pulmonary function tests (PFTs) performed more than 90 days apart were identified using our ILD registry. The electronic medical record was used to extract pertinent clinical data. Vital status was determined by review of medical records and telephone communication. Weight loss was assessed using BMI calculated from height and weight reported on serial PFT reports, which are collected per institutional protocol. All available PFTs performed within 48 months of ILD centre evaluation were included for review.

Longitudinal change in BMI was assessed using a maximum likelihood linear mixed effects model with time aligned to 3-month intervals. Baseline BMI, ILD diagnosis, gender age physiology (GAP) score [7], race, smoking history, anti-fibrotic exposure and immunosuppressant exposure were included as fixed effect variables to adjust for potential confounders of weight change over time, with intercept term used to account for random effects. Mortality risk was assessed using Poisson regression with a robust variance estimator [8] and survival time was assessed and plotted using the log rank test and Kaplan-Meier estimator, respectively. BMI was modelled as a continuous measure and binary variable stratified by greater or less than $5 \%$ mean yearly decline. Statistical significance was defined as $\mathrm{p}<0.05$. All statistical analyses were performed using Stata (Release 14; StataCorp, College Station, TX, USA).

Of 291 patients screened, 225 met inclusion criteria. The mean age was 70 years and a majority were male (56\%), white (74\%) and reported a history of smoking (56\%). The mean cohort BMI was 28.2, with 5\% underweight, $15 \%$ normal weight, $41 \%$ overweight and $36 \%$ obese. The mean percentage predicted forced vital capacity (FVC) and diffusing capacity of the lung for carbon monoxide (DLCO) were $72 \%$ and $54 \%$, respectively. A majority of patients carried a diagnosis of IPF (33\%), followed by CTD-ILD (31\%), CHP (19\%) and U-ILD (17\%).

Forty-eight percent $(n=108)$ of patients experienced $\geqslant 1 \%$ yearly BMI decline and $18 \%(n=40)$ experienced $\geqslant 5 \%$ yearly BMI decline during the study period. The estimated yearly decline in BMI was similar across ILD subtypes (IPF: $0.12 \mathrm{~kg} \cdot \mathrm{m}^{-2}$ (95\% CI 0.07-0.17), p<0.001; CTD-ILD: $0.08 \mathrm{~kg} \cdot \mathrm{m}^{-2}$ (95\% CI 0.01-0.14), $\mathrm{p}=0.03$; CHP: $0.11 \mathrm{~kg} \cdot \mathrm{m}^{-2}$ (95\% CI $\left.-0.07-0.28\right), \mathrm{p}=0.23$; and U-ILD: $0.14 \mathrm{~kg} \cdot \mathrm{m}^{-2}$ (95\% CI $0.03-0.25$ ), $\mathrm{p}=0.01$ ) (figure 1a), but did not reach statistical significance in the CHP cohort. Baseline BMI was

@ERSpublications

Weight loss in patients with ILD was associated with increased mortality risk in those with IPF and U-ILD http://ow.ly/Nk0D30l2Apm

Cite this article as: Pugashetti J, Graham J, Boctor $\mathrm{N}$, et al. Weight loss as a predictor of mortality in patients with interstitial lung disease. Eur Respir J 2018; 52: 1801289 [https://doi.org/10.1183/ 13993003.01289-2018]. 

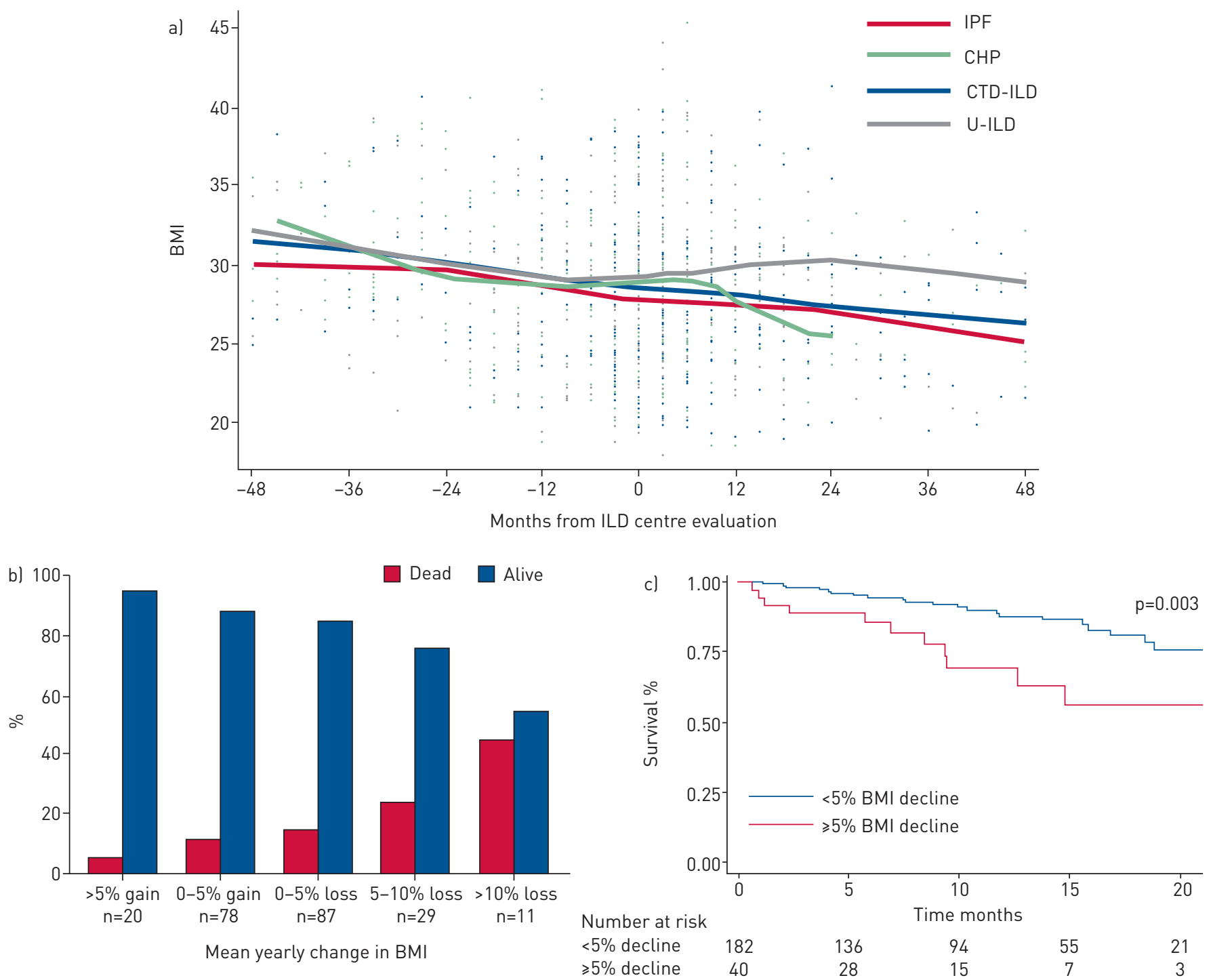

FIGURE 1 Mean body mass index (BMI) change over time among interstitial lung disease (ILD) subtypes (a); the proportion of patients surviving with each $5 \%$ yearly change in BMI (b); and survival time stratified by BMI decline, with those experiencing $\geqslant 5 \%$ yearly BMI decline displaying significantly worse survival compared to those with $<5 \%$ yearly BMI decline ( $p=0.003$ ) (c). IPF: idiopathic pulmonary fibrosis; CHP: chronic hypersensitivity pneumonitis; CTD-ILD: connective tissue disease-associated ILD; U-ILD: unclassifiable ILD.

marginally associated with differential BMI decline, with obese individuals demonstrating the largest yearly decline.

In outcome analysis, there was no association between baseline BMI and mortality, but longitudinal BMI decline did correlate with increasing mortality (figure $1 \mathrm{~b}$ ). Each $1 \%$ yearly decline in BMI was associated with a 5\% increase in mortality risk in unadjusted analysis (relative risk 1.05 (95\% CI 1.03-1.08), $\mathrm{p}<0.001$ ), which persisted after multivariable adjustment (relative risk 1.04 (95\% CI 1.01-1.06), p=0.004). These findings were driven primarily by patients with IPF (relative risk 1.06 (95\% CI 1.01-1.10), $\mathrm{p}=0.008$ ) and U-ILD (relative risk 1.09 (95\% CI 1.0-1.2), $\mathrm{p}=0.05$ ), as no association was observed in the CTD-ILD and CHP cohorts.

Survival time was significantly better in those with $<5 \%$ yearly BMI decline when compared to those with $\geqslant 5 \%$ yearly BMI decline (figure $1 \mathrm{c})(\mathrm{p}=0.003)$. Those with $\geqslant 5 \%$ yearly BMI decline had two-fold higher risk of death compared to those with $<5 \%$ yearly BMI decline (relative risk 2.41 (95\% CI 1.31-4.44), $\mathrm{p}=0.005)$ in unadjusted analysis, which persisted after multivariable adjustment (relative risk 2.0 (95\% CI $1.08-3.71$ ), $\mathrm{p}=0.03$ ). These findings were again driven primarily by patients with IPF (relative risk 3.1 (95\% CI 1.08-8.88), $\mathrm{p}=0.03$ ) and U-ILD (relative risk 9.21 (95\% CI 1.18-72.0), $\mathrm{p}=0.03$ ), as no association was observed in the CTD-ILD and CHP cohorts. 
In this study we found that weight loss is common among patients with ILD and is associated with an increased risk of death in those with IPF and U-ILD. Just as pulmonary function decline is a hallmark of progressive ILD [9-11], our findings suggest that weight loss may also serve as a longitudinal marker of ILD progression. To our knowledge, this study is the first to explore the clinical implications of weight loss amongst patients with ILD.

While obesity is a well-recognised risk factor for cardiovascular and metabolic conditions, unintentional weight loss is a well-described phenomenon in chronic illnesses such as emphysema and congestive heart failure $[12,13]$. It has been proposed that low BMI may be a determinant of poor survival in those with emphysema due to respiratory muscle weakness, impaired gas exchange and impaired immune response [14]. Similar phenomena may be at play in patients with ILD.

Our observed association between BMI decline and mortality risk in IPF and U-ILD, but not CTD-ILD and CHP, suggests that progressive disease may manifest differently in fibrotic-predominant disease compared to mixed inflammatory/fibrotic disease. However, the small sample size within each ILD subtype may have limited our ability to detect this association in those with CTD-ILD and CHP.

We did not find an association between baseline BMI and survival in our analysis; however, a previous investigation by ALAKHRAs et al. [6] showed that a higher baseline BMI was associated with improved outcomes, while KONDOH et al. [15] found that higher BMI was associated with increased acute exacerbation risk. The conflicting results of these investigations, along with our findings, leave it unclear as to the extent to which baseline BMI influences outcomes in this patient population.

Our study has several limitations. First, its retrospective nature allows for assessment of association but not causation. Secondly, the generalisability of our results may be limited given that our study was single centre and performed in patients somewhat older than other reported ILD populations. Thirdly, few patients had complete PFT data, resulting in a large number of missing time points for each patient. Finally, it was not possible to systematically ascertain whether the observed weight loss was unintentional, but the overwhelming majority of patients did not endorse intentional weight loss on chart review.

Weight loss is a common finding among patients with ILD and appears to be a marker of disease progression in those with IPF and U-ILD. Further research is needed to elucidate the biology underpinning this observation and to determine whether this finding is generalisable to other ILD cohorts. Finally, an assessment of whether nutritional augmentation in those with ILD experiencing ongoing weight loss modulates disease course is warranted.

Janelle Pugashetti ${ }^{1}$, Julia Graham ${ }^{1}$, Noelle Boctor ${ }^{1}$, Cesar Mendez ${ }^{2}$, Elena Foster ${ }^{2}$, Maya Juarez ${ }^{2}$, Richart Harper ${ }^{2}$, Brian Morrissey $^{2}$, Michael Kadoch ${ }^{3}$ and Justin M. Oldham ${ }^{2}$

${ }^{1}$ Dept of Internal Medicine, University of California at Davis, Sacramento, CA, USA. ${ }^{2}$ Division of Pulmonary, Critical Care and Sleep Medicine, University of California at Davis, Sacramento, CA, USA. ${ }^{3}$ Dept of Radiology, University of California at Davis, Sacramento, CA, USA.

Correspondence: Justin Oldham, Division of Pulmonary, Critical Care and Sleep Medicine, University of California at Davis, 4150 V St, Suite 4300, Sacramento, CA 95817, USA. E-mail: joldham@ucdavis.edu

Received: May 282018 | Accepted after revision: July 142018

Conflict of interest: R. Harper reports research support from Sacramento VA Medical Center, during the conduct of the study. M. Kadoch reports personal fees for speaking from Boehringer Ingelheim, outside the submitted work. J.M. Oldham reports personal fees from Boehringer Ingelheim and Genentech, outside the submitted work.

Support statement: This study was funded by a grant from the National Heart Lung and Blood Institute (K23HL138190). Funding information for this article has been deposited with the Crossref Funder Registry.

\section{References}

Fischer A, du Bois R. Interstitial lung disease in connective tissue disorders. Lancet 2012; 380: 689-698.

Selman M, Pardo A, King TE Jr. Hypersensitivity pneumonitis: insights in diagnosis and pathobiology. Am J Respir Crit Care Med 2012; 186: 314-324.

3 Raghu G, Collard HR, Egan JJ, et al. An official ATS/ERS/JRS/ALAT statement: idiopathic pulmonary fibrosis: evidence-based guidelines for diagnosis and management. Am J Respir Crit Care Med 2011; 183: 788-824.

4 Ryerson CJ, Urbania TH, Richeldi L, et al. Prevalence and prognosis of unclassifiable interstitial lung disease. Eur Respir J 2013; 42: 750-757.

5 Ryerson CJ, Vittinghoff E, Ley B, et al. Predicting survival across chronic interstitial lung disease: the ILD-GAP model. Chest 2014; 145: 723-728.

6 Alakhras M, Decker PA, Nadrous HF, et al. Body mass index and mortality in patients with idiopathic pulmonary fibrosis. Chest 2007; 131: 1448-1453.

7 Ley B, Ryerson CJ, Vittinghoff E, et al. A multidimensional index and staging system for idiopathic pulmonary fibrosis. Ann Intern Med 2012; 156: 684-691. 
8 Zou G. A modified poisson regression approach to prospective studies with binary data. Am J Epidemiol 2004; 159: 702-706.

9 Schmidt SL, Tayob N, Han MK, et al. Predicting pulmonary fibrosis disease course from past trends in pulmonary function. Chest 2014; 145: 579-585.

10 Zappala CJ, Latsi PI, Nicholson AG, et al. Marginal decline in forced vital capacity is associated with a poor outcome in idiopathic pulmonary fibrosis. Eur Respir J 2010; 35: 830-836.

11 Gimenez A, Storrer K, Kuranishi L, et al. Change in FVC and survival in chronic fibrotic hypersensitivity pneumonitis. Thorax 2018; 73: 391-392.

12 Prescott E, Almdal T, Mikkelsen KL, et al. Prognostic value of weight change in chronic obstructive pulmonary disease: results from the Copenhagen City Heart Study. Eur Respir J 2002; 20: 539-544.

13 Anker SD, Negassa A, Coats AJ, et al. Prognostic importance of weight loss in chronic heart failure and the effect of treatment with angiotensin-converting-enzyme inhibitors: an observational study. Lancet 2003; 361: 1077-1083.

14 Landbo C, Prescott E, Lange $\mathrm{P}$, et al. Prognostic value of nutritional status in chronic obstructive pulmonary disease. Am J Respir Crit Care Med 1999; 160: 1856-1861.

15 Kondoh Y, Taniguchi H, Katsuta T, et al. Risk factors of acute exacerbation of idiopathic pulmonary fibrosis. Sarcoidosis Vasc Diffuse Lung Dis 2010; 27: 103-110. 